\title{
Edge-to-edge repair for prevention and treatment of mitral valve systolic anterior motion
}

\author{
Patrick O. Myers, MD, ${ }^{\mathrm{a}}$ Zain Khalpey, MD, PhD, ${ }^{\mathrm{a}}$ Ann M. Maloney, ${ }^{\mathrm{a}}$ Derek R. Brinster, $\mathrm{MD},{ }^{\mathrm{c}}$ \\ Michael N. D'Ambra, MD, ${ }^{\mathrm{b}}$ and Lawrence H. Cohn, MD ${ }^{\mathrm{a}}$
}

\begin{abstract}
Background: The edge-to-edge technique has been proposed to prevent systolic anterior motion (SAM) of the mitral valve. There is limited clinical data available on outcomes of this technique for this indication. We reviewed the midterm results of this technique for SAM prevention and treatment.
\end{abstract}

\begin{abstract}
Methods: A total of 2226 patients had mitral valve repair between 2000 and 2011, 1148 of which were for myxomatous mitral regurgitation. Beginning in 2000, predictability of postrepair SAM based on the prebypass, intraoperative transesophageal echocardiogram arose in our program. The edge-to-edge technique was used in 65 patients $(5.7 \%)$ for SAM management, in 53 patients preemptively for transesophageal echocardiogrambased SAM prediction, and in 12 patients for postrepair SAM treatment.
\end{abstract}

Results: There was no operative mortality. Postoperative mitral regurgitation was significantly improved in all patients compared with the preoperative grade $(P<.001)$. SAM was completely eliminated, the mean mitral regurgitation grade in the postoperative period was $0.7 \pm 0.9$, and the mean transmitral gradient was $1.3 \pm$ $2.2 \mathrm{~mm} \mathrm{Hg}$. During a mean follow-up of 26 months, 1 patient in the SAM treatment group presented late recurrence of SAM and no patients developed mitral stenosis (mean transmitral gradient, $2.0 \pm 2.6 \mathrm{~mm} \mathrm{Hg} ; P=.12$ ). Without SAM prediction and preemptive edge-to-edge technique, the expected rate of SAM would have been $5.7 \%$; however, the observed rate was $1 \%$ (12 of 1148 patients).

Conclusions: Initiating an expectation for prebypass SAM prediction, combined with a surgical SAM prevention strategy, resulted in a reduced prevalence of SAM compared with our model of observed to-expected-ratios and to published norms. (J Thorac Cardiovasc Surg 2013;146:836-40)

In mitral valve (MV) repair for myxomatous prolapsed valves, there is the potential for creating systolic anterior motion (SAM) of the anterior mitral leaflet and consequent obstruction of the left ventricular outflow tract (LVOT), which can add significant morbidity and the need for a second exposure to cardiopulmonary bypass (CPB). ${ }^{1,2}$

Although the standard MV repair techniques, including quadrangular resection and sliding plasty to reduce the height of the posterior leaflet and ring annuloplasty, are usually sufficient to eliminate regurgitation and minimize the risk of SAM, it can still occur after repair. We previously reported our initial experience in using the edge-to-edge (E2E) technique in preventing SAM in patients at high risk based on prebypass pre-MV repair echocardiographic criteria or when it developed after MV repair. ${ }^{3}$ Because of

From the Division of Cardiac Surgery, ${ }^{\mathrm{a}}$ and Division of Cardiac Anesthesia, ${ }^{\mathrm{b}}$ Brigham and Women's Hospital and Harvard Medical School, Boston, Mass; and Virginia Commonwealth University Medical Center and Medical College of Virginia, ${ }^{c}$ Richmond, Va.

Disclosures: Authors have nothing to disclose with regard to commercial support.

Received for publication April 13, 2012; revisions received June 8, 2012; accepted for publication July 26, 2012; available ahead of print Sept 13, 2012.

Address for reprints: Lawrence H. Cohn, MD, Division of Cardiac Surgery, Brigham and Women's Hospital and Harvard Medical School, 75 Francis St, Boston, MA 02115 (E-mail: lhcohn@partrners.org).

$0022-5223 / \$ 36.00$

Copyright (c) 2013 by The American Association for Thoracic Surgery

http://dx.doi.org/10.1016/j.jtcvs.2012.07.051 this experience, during 2000 we initiated an expectation that intraoperative, prebypass transesophageal echocardiography (TEE) examinations would include a SAM prediction, which if repaired would lead to a very low prevalence of postrepair SAM.

This report expands on a previous study focusing on our early and medium-term experience with the E2E technique in MV repair in patients at high risk of SAM based on known preoperative assessment of SAM potential, or SAM after MV repair.

\section{METHODS}

\section{Study Design}

This study is a retrospective review of all patients who underwent surgical MV repair between 2000 and 2011 at our institution, in whom E2E was used to manage SAM. The study was approved by the Brigham and Women's Hospital Institutional Review Board, and patient consent was waived.

The primary outcome measure was the recurrence of SAM. Secondary outcomes were development of mitral stenosis, the need for MV reoperation, or significant mitral regurgitation.

\section{Surgical Technique}

All patients undergoing MV repair had intraoperative pre-CPB TEE and the MV was evaluated for anatomic causes of mitral regurgitation and the potential to develop SAM after the repair. The operative approach to MV repair was through a median sternotomy, which was limited to a lower hemisternotomy in isolated MV repairs, and through the left atrium after establishing $\mathrm{CPB}$. The valve was inspected intraoperatively to confirm the 


\author{
Abbreviations and Acronyms \\ A2 = middle scallop of the anterior mitral leaflet \\ $\mathrm{CPB}=$ cardiopulmonary bypass \\ $\mathrm{E} 2 \mathrm{E}=$ edge-to-edge \\ LVOT $=$ left ventricular outflow tract \\ $\mathrm{MV}=$ mitral valve \\ SAM $=$ systolic anterior motion \\ TEE = transesophageal echocardiography
}

echocardiographic findings and to identify any additional lesions. A variety of techniques were used to achieve a competent MV before using the E2E to manage SAM. A majority of patients underwent a posterior leaflet quadrangular resection with a modified sliding annuloplasty or folding valvuloplasty of a prolapsing posterior leaflet. ${ }^{4}$ All but 1 patient received a prosthetic annuloplasty ring. The E2E repair was used in 2 principal circumstances to manage SAM: to prevent SAM in patients deemed at high risk of developing SAM after MV repair, based on intraoperative prerepair TEE assessment of the mitral valve before CPB was instituted, and to treat SAM in patients in whom SAM was identified by TEE after initial termination of CPB after MV repair. The E2E stitch was placed to prevent SAM of the anterior mitral leaflet in the LVOT at the coaptation point of middle scallop of the anterior mitral leaflet (A2) and middle scallop of the posterior mitral leaflet. Mitral valve calipers were used to ensure that each of the 2 residual orifice areas was $>2 \mathrm{~cm}^{2}$, as described previously. ${ }^{3}$ The E2E stitch was a 2-0 multifilament suture placed in a figure of 8 suture mode from anterior to posterior leaflet.

Patients who had SAM after MV repair that was not satisfactorily responsive to the usual medical maneuvers (ie, volume resuscitation, beta blockade, and avoiding inotropes) were managed surgically by a second bypass run and placement of an E2E stitch.

\section{Prediction of SAM Potential}

The MV was determined to have SAM potential based on both preoperative TEE exam and surgical assessment upon direct inspection. Diagnostic elements contributing to the prediction of SAM consisted of 1 or more of the following: the presence of SAM in the prebypass period; interaction between the anterior leaflet and the subvalvular apparatus using the Maslow semiquantitative estimation of SAM potential ${ }^{5}$ (coaptation-septal distance $\leq 25 \mathrm{~mm}$ increases risk of SAM); asymmetry of the anterior leaflet, in which the anterolateral portion of A2 is larger than the posteromedial A2 (this measurement was reported previously. ${ }^{3}$ Asymmetry measured on TEE was validated by direct surgical measurement of anterior leaflet lengths using a modified MV sizer [see Figure 1]. Asymmetry [lateral A2 height minus medial A2 height $\geq 5 \mathrm{~mm}$ ] led to prediction of moderate-to-high risk of SAM with risk proportional to the degree of asymmetry); insertion location of A2 secondary chords, also reported previously $^{3}$; and degree of override of the mitral annulus over the LVOT (override of A2 over LVOT $>20 \%$ increases SAM risk in proportion to percentage override. Percentage override and the aorto/mitral angle measurement assess the same anatomical condition).

The cardiac anesthesiologist reported a high, moderate, or low potential for SAM to the surgeon before initiation of CPB. Criteria used for combined TEE and surgical assessment of significant SAM potential are summarized in Table 1. In addition to optimizing the height of middle scallop of the posterior mitral leaflet and sizing the annuloplasty band based on the height of the A2 segment from annulus to leaflet edge, surgical modifications mitigating high SAM risk included midline or offset E2E suture (Figure 2). E2E offset was guided by TEE and/or surgical evidence of A2 asymmetry.

\section{Statistical Analysis}

Statistical analyses were performed with SPSS software (2010, version 19; SPSS Inc, Chicago, Ill). Data are presented as mean \pm standard deviation. Continuous variables were analyzed with the Student $t$ test, or the related samples Wilcoxon signed rank test when appropriate, and categorical variables using the $\chi^{2}$ test or the Fisher exact test when appropriate. Actuarial estimates were calculated using the Kaplan-Meier method and differences between curves assessed by the log-rank test. The prevalence of E2E used for prevention or treatment SAM in the entire cohort of myxomatous MV repairs was considered as the expected rate of SAM; the prevalence of E2E used for SAM treatment in the same cohort of patients was considered as the observed rate of intraoperative SAM; finally, the prevalence of E2E for SAM treatment or late development of SAM was used as the observed rate of overall SAM. Differences between expected and observed rates of SAM were compared using the Fisher exact test. All statistical tests were 2-tailed.

\section{RESULTS}

\section{Patient Characteristics}

A total of 2226 patients had MV repair between October 2000 and July 2011 at our institution, 1148 of which were for myxomatous mitral regurgitation. The E2E stitch was used in 65 patients $(5.7 \%)$ for SAM management, in 53 patients for prevention of postrepair high risk of SAM, and in 12 patients for postrepair SAM treatment. Mean age at repair was $57 \pm 11$ years and $36(55.4 \%)$ were men. The mean New York Heart Association Functional Class at admission was $1.8 \pm 0.8$, and mean left ventricular ejection fraction was $61 \% \pm 11 \%$. Patient baseline characteristics are summarized in Table 2. Based on our SAM prediction algorithm, the expected rate of SAM was estimated at $5.7 \%$ (65 of 1148 patients). With implementation of prebyass SAM prediction combined with surgical SAM prevention, the observed rate of intraoperative SAM was reduced to $1 \%(12$ of 1148$)(P<.0001)$.

\section{Operative Characteristics}

Operative details are summarized in Table 3 . All but $1 \mathrm{pa}-$ tient had ring annuloplasty $(98.5 \%)$, mostly with the Cosgrove-Edwards band (Edwards Lifesciences LLC, Irvine, Calif). The mean ring size was $36.1 \pm 2.2 \mathrm{~mm}, 2.7$ $\mathrm{mm}$ larger in the SAM prevention group compared with the SAM treatment group $(P<.001)$. Not surprisingly, patients in the SAM treatment group had longer CPB and aortic cross-clamp times, by 33 and 19 minutes, respectively ( $P=.005$ and $P=.02$, respectively), due to the second bypass run after MV repair to correct SAM.

\section{Outcomes}

There was no operative mortality in either E2E groups, and 6 patients $(0.5 \%)$ died in the overall myxomatous MV repair cohort. Early postoperative mitral regurgitation was significantly improved in all patients compared with the preoperative grade, from a mean mitral regurgitation grade of $3.9 \pm 0.3$ (severe) prerepair to $0.8 \pm 0.9$ (none to trivial $)(P<.001)$. The mean transmitral gradient was 


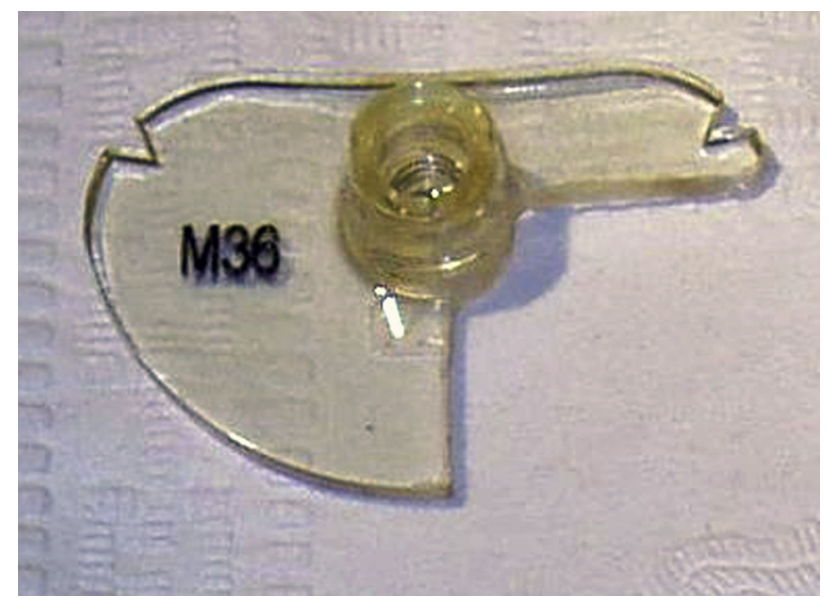

FIGURE 1. Modified Edwards mitral sizer (Edwards Lifesciences LLC, Irvine, Calif). This device allows for precise measurement of middle scallop of the anterior mitral leaflet annulus to leaflet edge for the lateral and the medial aspects of the anterior mitral leaflet segment. It also facilitates assessment of the degree of asymmetry of anterior mitral leaflet.

increased, from a mean of $0.1 \pm 0.5 \mathrm{~mm} \mathrm{Hg}$ to $1.3 \pm 2.2 \mathrm{~mm}$ $\mathrm{Hg}(P=.002)$. No patient had evidence of SAM on postoperative imaging.

The 12 patients who underwent a second cross-clamp were deemed by the operating surgeon to not be at a high risk of SAM, based on the integrated TEE and surgical assessment of the valve. On review of the TEEs and records, among these 12 patients, 1 patient had high TEE-predicted SAM potential but was not confirmed by surgical assessment; the SAM potential was not noted on the anesthesia record in the remaining 11 patients. On re-review of the TEE images, the SAM potential was noted to be high in 9

TABLE 1. Criteria for determination of significant systolic anterior motion (SAM) potential

\begin{tabular}{ll}
\hline \multicolumn{1}{c}{ Assessment } & \multicolumn{1}{c}{ Criteria } \\
\hline TEE criteria & Prerepair SAM \\
& C-sept distance $\leq 25 \mathrm{~mm}$ \\
& Anterolateral A2 height - posteromedial \\
A2 height $\geq 5 \mathrm{~mm}$ \\
Insertion location of A2 secondary chords \\
A2 override over the LVOT $>20 \%$ \\
Height of A2 from annulus to leaflet edge \\
using modified Edwards mitral sizer, \\
including assessment of asymmetry of \\
lateral and medial portions of A2 \\
After placement of annuloplasty band, \\
remeasurement of A2 height to determine \\
if $>7$ to 8 mm of anterior leaflet was below \\
the coaptation line \\
P2 height reducible to 10 mm
\end{tabular}

$T E E$, Transesophageal echocardiography; SAM, systolic anterior motion; $C$-sept, coaptation to septum; $A 2$, middle scallop of the anterior mitral leaflet; $L V O T$, left ventricular outflow tract; $P 2$, middle scallop of the posterior mitral leaflet. *See Figure 2.

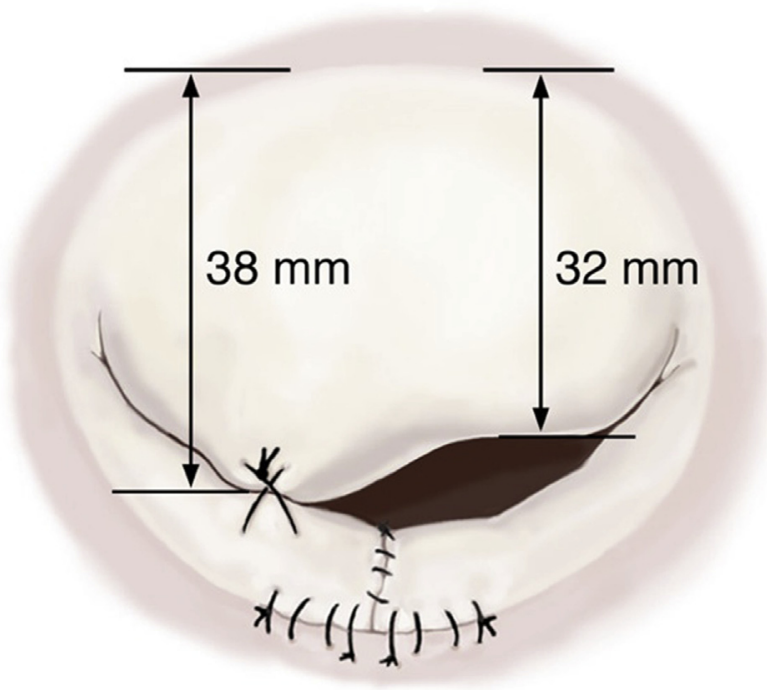

FIGURE 2. Example of an offset placement of an edge-to-edge suture. It is important to only perform this offset in the face of asymmetry of the middle scallop of the anterior mitral leaflet segment. Offset of edge-to-edge in a symmetric anterior mitral leaflet may result in development of late mitral regurgitation.

patients and moderate in 2 patients. It is impossible to determine retrospectively if the SAM potential was not assessed fully in these 11 patients. The operating surgeon evaluated

TABLE 2. Baseline patient characteristics

\begin{tabular}{|c|c|c|c|}
\hline & $\begin{array}{l}\text { SAM prevention } \\
\quad(\mathbf{n}=\mathbf{5 3})\end{array}$ & $\begin{array}{l}\text { SAM treatment } \\
(\mathrm{n}=12)\end{array}$ & $\boldsymbol{P}$ \\
\hline Man & $30(56.6)$ & $6(50)$ & 46 \\
\hline Age (y) & $57.3 \pm 11.6$ & $56.3 \pm 11.6$ & .79 \\
\hline $\operatorname{LVEF}(\%)$ & $61.5 \pm 8.4$ & $60.6 \pm 18.4$ & .88 \\
\hline \multicolumn{4}{|l|}{ NYHA Functional Class } \\
\hline I & $22(41.5)$ & $4(33.3)$ & .80 \\
\hline II & $21(39.6)$ & $6(50)$ & .80 \\
\hline III & $10(18.9)$ & $2(16.7)$ & .80 \\
\hline Smoking history & $12(22.6)$ & $2(16.7)$ & .46 \\
\hline Diabetes & $1(1.9)$ & $0(0)$ & .81 \\
\hline Hypercholesterolemia & $8(15.1)$ & $3(25)$ & 46 \\
\hline Prior stroke & $1(1.9)$ & $0(0)$ & 1 \\
\hline Peripheral vascular disease & $0(0)$ & $0(0)$ & 1 \\
\hline Cerebrovascular disease & $1(1.9)$ & $0(0)$ & 1 \\
\hline \multicolumn{4}{|l|}{ Chronic lung disease } \\
\hline None & $39(92.9)$ & $9(90)$ & .09 \\
\hline Mild & $3(7.1)$ & $0(0)$ & .09 \\
\hline Moderate & $0(0)$ & $1(10)$ & .09 \\
\hline \multicolumn{4}{|l|}{ Mitral regurgitation } \\
\hline Mild & $0(0)$ & $0(0)$ & 1 \\
\hline Moderate & $5(9.4)$ & $1(8.3)$ & 1 \\
\hline Severe & $48(90.6)$ & $11(91.7)$ & 1 \\
\hline Systolic anterior motion & $0(0)$ & $1(8.3)$ & .19 \\
\hline
\end{tabular}

All data are presented as mean \pm standard deviation or number (\%). $L V E F$, Left ventricular ejection fraction; NYHA, New York Heart Association; SAM, systolic anterior motion. 
TABLE 3. Operative details

\begin{tabular}{|c|c|c|c|}
\hline & \multicolumn{3}{|c|}{ SAM prevention SAM treatment } \\
\hline Ring annuloplasty & $53(100)$ & $11(91.7)$ & .19 \\
\hline Cosgrove-Edwards* & $50(94.3)$ & $8(66.7)$ & .002 \\
\hline Carpentier-Edwards classic* & $1(1.9)$ & $0(0)$ & .002 \\
\hline Carpentier-Edwards Physio* & $1(1.9)$ & $0(0)$ & .002 \\
\hline Carbomedics AnnuloFlex $\dagger$ & $0(0)$ & $3(25)$ & .002 \\
\hline Medtronic Duran $\ddagger$ & $1(1.9)$ & $0(0)$ & .002 \\
\hline No annuloplasty & $0(0)$ & $1(8.3)$ & .002 \\
\hline Ring size & $36.7 \pm 2.0$ & $34 \pm 1.8$ & $<.001$ \\
\hline \multicolumn{4}{|l|}{ Leaflet repairs } \\
\hline $\begin{array}{l}\text { PL segment resection and } \\
\text { modified sliding plasty }\end{array}$ & $29(54.7)$ & $7(58.3)$ & 1 \\
\hline PL foldoplasty & $21(39.6)$ & $4(33.3)$ & .75 \\
\hline Commissuroplasty & $13(24.5)$ & $1(8.3)$ & .44 \\
\hline PL cleft repair & $5(9.4)$ & $1(8.3)$ & 1 \\
\hline AL GoreTex§ neochordae & $0(0)$ & $1(8.3)$ & .19 \\
\hline \multicolumn{4}{|l|}{ Concomitant procedures } \\
\hline Aortic valve replacement & $11(20.8)$ & $3(25)$ & .85 \\
\hline Aortic valve repair & $1(1.9)$ & $0(0)$ & .85 \\
\hline CABG & $4(7.5)$ & $1(8.3)$ & 1 \\
\hline Tricuspid valve repair & $4(7.5)$ & $0(0)$ & .58 \\
\hline $\begin{array}{l}\text { Cardiopulmonary bypass time } \\
\quad(\min )\end{array}$ & $123.9 \pm 33.7$ & $156.8 \pm 31.3$ & .005 \\
\hline Aortic cross-clamp time (min) & $86.1 \pm 23.1$ & $104.8 \pm 21.9$ & .02 \\
\hline
\end{tabular}

Data are presented as mean \pm standard deviation or number (\%). SAM, Systolic anterior motion; $P L$, posterior mitral leaflet; $A L$, anterior mitral leaflet; $C A B G$, coronary artery bypass graft surgery. *Edwards Lifesciences, Irvine, Calif. †Sorin Group, Milano, Italy. $\ddagger$ Medtronic, Minneapolis, Minn. §W.L. Gore Associates, Newark, Del.

the valve and considered the SAM potential to be low in all of these 12 patients and did not perform an E2E repair. If the E2E had been used in all patients with a high SAM potential by TEE assessment, the observed rate of SAM could have been further reduced to 2 of $1148(0.2 \%)$.

Late results are summarized in Table 4. During a mean follow-up of $26.4 \pm 24.7$ months (range, 1 month to 6.6 years), there were no late reoperations. One patient in the SAM treatment group developed late SAM, and as of this report is being managed medically. In terms of function of the MV, 3 patients $(6.7 \%)$ developed moderate mitral regurgitation at late follow-up, all in the SAM prevention group. The freedom from SAM or MV reoperation (see Figure 3) was $100 \%$ in the SAM prevention group up to 6.6 years, and in the SAM treatment group freedom from SAM or MV reoperation was $100 \%$ at 1 year and $80 \% \pm 18 \%$ at

TABLE 4. Late results

\begin{tabular}{lccc}
\hline & $\begin{array}{c}\text { SAM prevention } \\
(\mathbf{n}=\mathbf{5 3})\end{array}$ & $\begin{array}{c}\text { SAM treatment } \\
(\mathbf{n}=\mathbf{1 2})\end{array}$ & $\boldsymbol{P}$ \\
\hline Follow-up (mo) & $27.3 \pm 26.2$ & $21.9 \pm 5.8$ & .46 \\
Systolic anterior motion & $0(0)$ & $1(8.3)$ & .17 \\
Transmitral gradient $(\mathrm{mm} \mathrm{Hg})$ & $2.1 \pm 2.6$ & $1.4 \pm 0.9$ & .53 \\
$\geq$ Moderate MR & $3(4)$ & $0(0)$ & 1 \\
\hline
\end{tabular}

Data are presented as mean \pm standard deviation or number (\%). SAM, Systolic anterior motion; $M R$, mitral regurgitation.

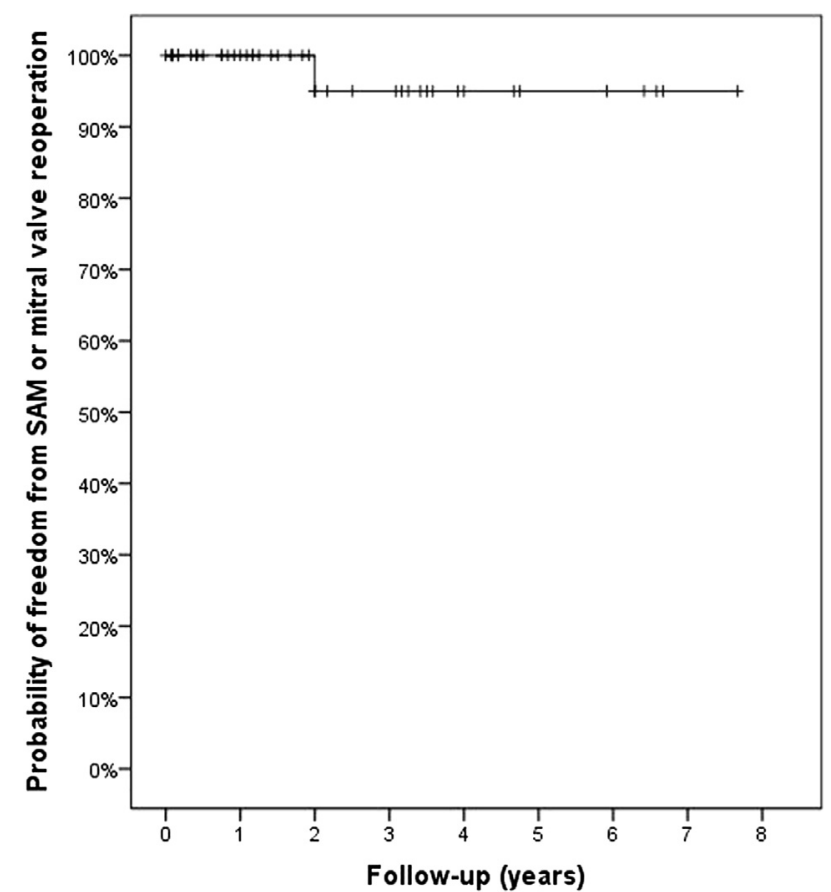

FIGURE 3. Kaplan-Meier analysis of freedom from recurrence of systolic anterior motion (SAM) and/or mitral valve reoperation.

2 and 4 years (log-rank test $P=.08$ ). Through implementation of prebyass SAM prediction combined with surgical SAM prevention, the observed rate of overall SAM was reduced to $1.0 \%(12$ of 1148$)(P<.0001)$.

\section{DISCUSSION}

Although multiple surgical techniques have been proposed to prevent SAM, it remains a challenging problem after MV repair. The overall prevalence of SAM has been reported to vary between $7 \%$ and $11 \% .^{6,7}$ Carpentier introduced quadrangular resection and sliding plasty to reduce the height of the posterior leaflet in an effort to reduce the risk of SAM after MV repair. ${ }^{8}$ We previously described folding valvuloplasty as a simple nonresectional method of reducing posterior leaflet height and avoiding $\mathrm{SAM}^{4}$; however, no formal evaluation of the incidence of SAM in this setting has been done. Triangular resection of the anterior leaflet has also been proposed. ${ }^{9}$

A majority of patients with postrepair SAM can be managed conservatively, with beta blockade and volume resuscitation. Factors that tend to increase the risk of SAM include the use of triangular resection of posterior leaflet prolapse without reducing the height of the posterior leaflet to $15 \mathrm{~mm}$ or sliding plasty, use of flexible and rigid complete rings that reduce the aortomitral angle, and excessive reduction of the mitral annulus, whereas treatment of anterior pathology with limited resection or neochordae tends to decrease the prevalence of SAM. ${ }^{6,9,10}$ 
Adams and colleagues ${ }^{11}$ proposed comprehensive and systematic algorithms for managing postrepair SAM, both intraoperatively and later, with excellent results. ${ }^{1}$ In their review of $785 \mathrm{MV}$ repairs, 52 patients $(6.6 \%)$ developed SAM while admitted to a hospital, and only 6 required surgical repeat repair. However, this approach proposes management strategies once SAM has developed, and does not propose a method of preventing it.

In our study, we show that E2E can safely be used as a surgical strategy to treat or prevent SAM, avoiding more complicated alternatives such as annuloplasty ring removal, downsizing, or anterior leaflet height shortening or neochordae. The risk of redeveloping late SAM despite the E2E within this patient population tended to be increased in patients with SAM diagnosed after repair. No patients developed significant stenosis secondary to the E2E repair. There were no technical failures of the E2E repair, which we attribute to using a multifilament, braided suture in a figure of 8 pattern, and not a monofilament.

Of patients who underwent myxomatous mitral valve repair, $5.7 \%$ were either found to have SAM after repair or were considered to be at high risk of SAM based on our prediction model. This rate is consistent with those reported previously in the literature, estimating between $7 \%$ and $11 \% .{ }^{6,7}$ However, by applying our preventive E2E based on prebypass SAM prediction, the actual rate of SAM postrepair was decreased to $1 \%$ (12 of 1148 procedures).

This is the largest study that looks specifically at E2E for management of SAM. Gillinov and colleagues ${ }^{12}$ first described using this technique in a single patient with a structurally normal MV with $3+$ mitral regurgitation and SAM after aortic root replacement. Mascagni and colleagues ${ }^{13}$ described using this technique to prevent postrepair SAM in 4 patients. We previously reported our early results in 20 patients. ${ }^{3}$ This study expands on these prior reports, providing longer follow-up, a larger patient population, and for the first time shows a not insignificant risk of recurrence of SAM despite E2E.

Our study is subject to all the limitations of a singleinstitution, retrospective study. Findings from the series may not be generalizable to other populations. Because this was not a randomized study, we could not control for all differences in the patient groups. Although this is a relatively large series of patients for these criteria, the number of patients in our study limits our ability to evaluate risk factors or to match cases to better control for differences between the 2 cohorts. The annuloplasty ring was significantly larger in the SAM prevention group, which potentially could reduce the risk of postrepair SAM independently of the E2E stitch, and confound the comparison between groups. In addition, patients received their surgeries across a 10-year span; clinical practice, patient characteristics, and comorbidities changed over time. Outcomes for remote cases may lack generalizability to future patient populations. Assessing the accuracy of our SAM prediction model is beyond the scope of our study, and only the echocardiograms of patients having had an E2E for SAM management were reviewed. Furthermore, follow-up was limited to the patients with E2E for SAM, not the entire cohort of 1148 myxomatous MV repair patients, and could potentially miss late-onset SAM in patients who did not require surgical management.

\section{CONCLUSIONS}

E2E can safely be used as a surgical strategy to treat or prevent SAM. The strategy is based on an expectation that it is possible to predict SAM, resulting a muchreduced prevalence of SAM compared with the norm when these predictions are combined with modifications of the surgical approach. Our study demonstrates that a preemptive, single-pump-run solution to the SAM problem is possible. The next steps will be to validate and optimize the SAM prediction methodology, and to continue to evolve the surgical modifications.

\section{References}

1. Lee KS, Stewart WJ, Lever HM, Underwood PL, Cosgrove DM. Mechanism of outflow tract obstruction causing failed mitral valve repair. Anterior displacement of leaflet coaptation. Circulation. 1993;88(5 Pt 2):II24-9.

2. Carpentier A. Cardiac valve surgery - the "French correction" J Thorac Cardiovasc Surg. 1983;86:323-37.

3. Brinster DR, Unic D, D'Ambra MN, Nathan N, Cohn LH. Midterm results of the edge-to-edge technique for complex mitral valve repair. Ann Thorac Surg. 2006; $81: 1612-7$.

4. Cevasco M, Myers PO, Elbardissi AW, Cohn LH. Foldoplasty: a new and simplified technique for mitral valve repair that produces excellent medium-term outcomes. Ann Thorac Surg. 2011;92:1634-7; discussion, 7-8.

5. Maslow AD, Regan MM, Haering JM, Johnson RG, Levine RA. Echocardiographic predictors of left ventricular outflow tract obstruction and systolic anterior motion of the mitral valve after mitral valve reconstruction for myxomatous valve disease. J Am Coll Cardiol. 1999;34:2096-104

6. Brown ML, Abel MD, Click RL, Morford RG, Dearani JA, Sundt TM, et al. Systolic anterior motion after mitral valve repair: is surgical intervention necessary? J Thorac Cardiovasc Surg. 2007;133:136-43.

7. Freeman WK, Schaff HV, Khandheria BK, Oh JK, Orszulak TA, Abel MD, et al. Intraoperative evaluation of mitral valve regurgitation and repair by transesophageal echocardiography: incidence and significance of systolic anterior motion. J Am Coll Cardiol. 1992;20:599-609.

8. Jebara VA, Mihaileanu S, Acar C, Brizard C, Grare P, Latremouille C, et al. Left ventricular outflow tract obstruction after mitral valve repair. Results of the sliding leaflet technique. Circulation. 1993;88(5 Pt 2):II30-4.

9. Grossi EA, Steinberg BM, LeBoutillier M 3rd, Ribacove G, Spencer FC, Galloway AC, et al. Decreasing incidence of systolic anterior motion after mitral valve reconstruction. Circulation. 1994;90(5 Pt 2):II195-7.

10. Sternik L, Zehr KJ. Systolic anterior motion of the mitral valve after mitral valve repair: a method of prevention. Tex Heart Inst J. 2005;32:47-9.

11. Varghese R, Anyanwu AC, Itagaki S, Milla F, Castillo J, Adams DH. Management of systolic anterior motion after mitral valve repair: an algorithm. $J$ Thorac Cardiovasc Surg. 2012;143(4 Suppl):S2-7.

12. Gillinov AM, Smedira NG, Shiota T. Use of the Alfieri edge-to-edge technique to eliminate left ventricular outflow tract obstruction caused by mitral systolic anterior motion. Ann Thorac Surg. 2004;78:e92-3.

13. Mascagni R, Al Attar N, Lamarra M, Calvi S, Tripodi A, Mebazaa A, et al. Edgeto-edge technique to treat post-mitral valve repair systolic anterior motion and left ventricular outflow tract obstruction. Ann Thorac Surg. 2005;79:471-3; discussion, 4 . 